deep hood or wire grid and should be as few as possible.

2. The lamp should be fitted with a good switch, in which the moving parts separate far enough to preclude all chance of arcing. A double pole type would be of advantage to ensure both electrodes being dead before they can be touched in the replacement of carbons, etc.

3. A well-fitting pair of goggles should always be worn. The use of goggles is so essential, especially with the small mercury arcs, that it would be an excellent thing if manufacturers agreed to mark all lamps intended for home use with the inscription, "Goggles must be worn when using this lamp." At present it is to be regretted that in a number of catalogues, etc., the artist has tried to express the pleasures of basking in artificial sunlight, but has omitted in his pictures the disfiguring goggles. The necessity of using goggles is mentioned in the catalogues, but a good illustration is often remembered when the written instructions have been mislaid. There are many satisfactory makes to be obtained, but, if there is any question, the only certain test is by spectrometer. A good pair of goggles should not transmit any ultra-violet radiation at all, not even of the longer wave-lengths.

The danger of falling asleep during the exposure is a perfectly definite one, as ultra-violet radiation often has that effect. In such a case it is possible for the patient to experience a severe blistering of the skin due to over-exposure, as this skin-burning does not appear until some time has elapsed. The simplest manner of guarding against this danger is to make it a rule always to stand up for an ultra. violet bath, and never to sit or lie down.

The most suitable place for the installation of these lamps is a matter for careful consideration. The bathroom, as has been pointed out elsewhere, is not the safest place, because of the danger of a bad shock due to the moisture present. On the other hand, it should be remembered that this radiation is a very powerful bleaching agent, and that the colours of fabrics, etc., will fade, and often the materials themselves perish, if exposed constantly to the rays.

The lamps reviewed here have been considered from the point of view of the man who wishes to have ultra-violet radiation available in his house as a tonic during the dark winter months. To use it for the treatment of any definite disease without the direction of a qualified physician is highly dangerous, for it must be realised that the irradiation used in medicine is often only a part of curative treatment. Further, there are conditions and diseases which can be made worse by ultraviolet radiation, and in spite of the enthusiastic pamphlets and catalogues which record its application in every human ill, it cannot be too strongly insisted that ultra-violet radiation is not a universal panacea.

\title{
The Ultra-Violet Transmission of Transparent Materials.
}

\section{By Dr. L. C. Martin.}

$\mathrm{W}^{\mathrm{H}}$ E may be forgiven for a little scepticism regarding the highly coloured reports which have been current during the last few years concerning the possibilities and effects of ultraviolet radiation. It must be admitted, however, that there appears to be a considerable measure of truth in such accounts; the physicist must assist in disentangling the errors and in weighing the evidence.

The shortest wave-length for which light is visible to the eye is roughly $0 \cdot 39 \mu$, but the spectrum of sunlight extends, although faintly in the end, to a wave-length of about $0 \cdot 295 \mu$ at sea-level; the cornea, the outer transparent coat of the eye, transmits down to about the sunlight limit. The 'lens' of the eye is more opaque; it will not transmit beyond $0.376 \mu$, and can be made to fluoresce by radiation of about this wave-length reaching it through the cornea. Severe inflammation of the conjunctiva can be caused by exposure to intense radiation of wave-lengths shorter than about $0 \cdot 305 \mu$. Physiological and germicidal actions occur with radiation of still shorter wave-lengths down to about $0 \cdot 21 \mu$, but beyond this point we soon reach the region $0 \cdot 193 \mu$ to $0 \cdot 185 \mu$, where air becomes practically opaque. The region of interest in ultraviolet transmission measurements for present purposes extends, then, roughly from $0.4 \mu$ to $0 \cdot 2 \mu$.

In connexion with the various sources for the production of ultra-violet radiation for clinical purposes, the transmission of the globes, screens, or containing vessels has to be studied; the transmission of window glass is also a special case. It may be added that the photometric study of the emission of the source is of no less importance. Naturally, the ultra-violet emission of many sources such as tungsten filament lamps, for which the familiar 'black-body' radiation laws are a sufficient guide, is extremely low in proportion to the total radiation. Much more energy, relatively speaking, is derived from arcs and sparks which give bright line spectra. The region $0 \cdot 4 \mu$ to $0 \cdot 2 \mu$ can be studied with the aid of fluorescence or photography. In some cases the absorption of media grows very rapidly with changing wavelength in a certain region; thus the simplest kind of information useful in some cases is the short wave-length limit of the transmission.

Quartz in the crystalline state is fairly transparent down to $0 \cdot 18 \mu$ in pieces $2 \mathrm{~cm}$. in thickness : the quartz spectrograph as made by the firms of Hilger and Bellingham and Stanley, in which the whole optical system is made in quartz, projects the entire visible and ultra-violet spectrum down to about $0 \cdot 185 \mu$ on a photographic plate; the substitution of a screen of uranium glass for the plate converts the instrument into an ultra-violet spectroscope in which the lines of the spectrum are seen by fluorescence. Wave-lengths can be given by a scale marked in the glass. An iron arc or 
tungsten arc gives a wealth of lines in the ultraviolet spectrum, so that if a piece of the substance under test has sufficiently good surfaces it may be held in the path of the light reaching the slit, when its ' limit of transmission' may become manifest by the darkening of the spectrum beyond a certain point. Such tests are useful for protective glasses or goggles. A compact ultra-violet spectroscope for hand use is made by Messrs. R. and J. Beck, Ltd.

Fluorite is one of the most transparent media for the ultra-violet (when pure it transmits down to $0 \cdot 125 \mu$ ), but it is difficult to obtain good specimens of any size, so that even its optical use is rather restricted; but this crystal, together with rocksalt and certain other crystals and gems, has important possibilities for scientific purposes.

Fused quartz is not doubly refracting, and is little less transparent than crystalline quartz; it has great resistance to heat, and its extremely high melting point makes it specially suitable for containing the mercury arc, one of the most useful sources of ultra-violet radiation. In spite of a great deal of research carried out for long periods, it has not yet been found possible to produce the material in a truly homogeneous state; there is always a residual structure showing small local variations of refractive index. Even though microscope lenses are made from it, they could be improved if the homogeneity were perfected.

The cost of using fused quartz for windows would be prohibitive, but since the range of transmission of glass can be made to include the whole sunlight range, it is unnecessary. Common soda glass is practically opaque beyond $0 \cdot 33 \mu$, but in Hovestadt's "Jena Glass" the increased ultra-violet transparency due to the use of barium is pointed out. Generally speaking, the transparency diminishes with increasing proportions of lead in the flint glasses. Researches in the effect of the composition of glass on the ultra-violet transparency have been made by Zschimmer, who found that boric oxide was very transparent. The use of sodium oxide in a glass decreased the transparency more than potassium oxide. Useful figures and curves are given by Schulz ("Das Glas," Munich; 1923. Kösel and Pustet) from the results of H. A. Kruss. Particulars are also given of the 'Uviol' Jena glass which has been most useful for spectroscopes and other purposes, since it transmits down to $0 \cdot 280 \mu$. Fritsch has published particulars of a glass made from calcium fluoride and boric oxide which is said to be transparent down to $0 \cdot 185 \mu$.

Comparatively recently, Messrs. Chance Bros. of Birmingham have brought out their 'Vita-glass' made to the specification of Lamplough. In a plate $2 \mathrm{~mm}$. thick this will still transmit 10 per cent. at $0 \cdot 272 \mu$. Also, the Corning Glass Works have announced a special ultra-violet transmitting glass (not yet available in large pieces) for which an even greater transparency is claimed.
The measurement of the transmission is usually made with the aid of the spectrograph. Half the slit is illuminated by light transmitted by the specimen, the other half by an exactly similar beam, the intensity of which can be diminished (in effect at least) in definite ratios by a special device. The spectrum is thus split into two; we find the wave-lengths at which the intensity has been diminished by the specimen to those ratios used for the above device, by comparing the densities of the two parts of each spectrum photograph. The action in the variable beam can be controlled by a polarisation device or by a rotating sector ; in the latter case it seems to be sufficiently accurate to assume that the reciprocity relation is valid for the plate. The optical arrangements for carrying out these tests have been so far standardised that they can be used without difficulty to obtain trustworthy results.

Special apparatus for ultra-violet spectrophotometry, for which greater speed and ease of working is claimed, has also been developed by $\mathrm{S}$. Judd Lewis. Mention must also be made of the photographic methods in which special non-selective 'wedges' are employed; such methods have been worked out (chiefly for the visible region) by Mees, Dobson, and others; in some cases they can be applied to the ultra-violet.

The durability of the new glasses referred to above can only be tested properly by experience. One important matter is their stability under the continuous action of ultra-violet radiation. Some glasses containing small amounts of manganese are well known to develop a pink tinge when used in globes round arc lamps; such action under exposure to intense radiation is likely seriously to prejudice the ultra-violet transmission, and effects of a similar kind should be looked for even in the absence of manganese. Naturally, the relation between the conditions of normal use and the conditions of the test would have to be carefully considered.

Important questions are likely to arise in connexion with the screening off of undesirable components of the radiation; it is possible that liquid 'filters' may become useful in such cases. Much information on recent work on the transmission of liquids is given in Luckiesh's useful book on "Ultra-violet Radiation," and recent work on the transmission of solutions has also been carried out by Dahm.

In conclusion, it may be said that photometric methods are so far developed that it should not be difficult to measure and to control the characteristics and intensity of any ultra-violet radiation used for medical purposes. In the somewhat arbitrary and experimental condition of present practice, it is not possible to be too precise and definite in the specification of physical conditions. 